Rev. Elev. Méd. vét. Pays trop., 1970, 23 (4) : 503-17

\title{
Pâturage aérien au Cameroun. Utilisation des ligneux par les bovins
}

\author{
par J. PIOT $\left(^{*}\right)$
}

\begin{abstract}
RESUME
L'auteur relate les observations effectuées dans les pâturages de Wakwa près de N'Gaoundéré tant sur les plants que sur des lots d'animaux au pâturage. La valeur bromatologique des espèces est donnée, ainsi que leur rendement et le comportement des troupeaux.

Des conséquences pratiques sont données concernant les possibilités de complémentation, le traitement des espèces ligneuses et la nécessité de laisser les animaux en permanence dans les parcs à exploiter.

En conclusion, l'auteur indique que la savane pastorale et la savane forestière, loin de s'opposer, sont complémentaires dans les conditions de l'élevage extensif actuel et souligne la nécessité du maintien d'un équilibre sylvo-pastoral convenable.
\end{abstract}

Il est connu que, dans les régions tropicales et équatoriales, le bétail consomme les feuilles et les petites branches des arbres et arbustes; l'émondage des acacias par les bergers et les chevriers pour l'affouragement de leurs troupeaux en saison sèche, est effectué couramment.

Si les recherches sur la flore des pâturages aériens, sont nombreuses, celles relatives à leur utilisation par les animaux et leur valeur bromatologique sont relativement rares.

Les observations relatées dans cet article ont été faites à Wakwa près de N'Gaoundéré dans les pâturages qui ont servi à l'étude précédemment publiée (13).

\section{EXPERIMENTATION}

Les observations ont été faites à la fois sur les plants et sur des lots d'animaux au pâturage. Des échantillons ont été prélevés sur les parties des plantes consommées habituellement par ces derniers, la détermination de la composition

(*) (I.E.M.V.T. Station fourragère de Wakwa, Cameroun'). chimique et de la valeur alimentaire a été effectuée sur des parties aliquotes des échantillons par R. RIVIERE, au laboratoire de nutrition, de l'IEMVT à Maisons-Alfort, suivant les méthodes classiques en la matière.

L'appétibilité a été notée dans un pâturage dont la flore avait été inventoriée.

Le comportement des troupeaux a été observé :

1. dans un pâturage sur sol granitique qui était brûlé régulièrement;

2. dans un pâturage sur sol granitique et basaltique rouge qui n'était pas brûlé, les animaux recevant une supplementation avec des feuilles de Daniellia oliveri.

\section{VALEUR BROMATOLOGIQUE}

a) Espèces appétées

La florule jointe en annexe de l'article "Végétaux ligneux et pâturages des savanes de l'Adamaoua » (13) donne à ce sujet toute précision quant à l'appétibilité des différentes espèces. 


\section{Non appétée (NA) \\ Peu appétée (PA) \\ Appétée \\ Très appétée \\ (TA)}

Il convient cependant d'insister sur quelques points particuliers.

Si certaines espèces sont appréciées de tous les animaux et en tous temps, d'autres peuvent ne l'être que par certaines bêtes, ce qui est lié à des différences de comportement causées soit par des dépravations du goût, soit par l'absence d'habitudes alimentaires. Enfin certaines espèces ne sont consommées qu'à certaines époques, c'est ainsi qu'en période de disette les animaux consomment les jeunes feuilles de Terminalia macroptera, ou de Syzygium guineense var. macroptera.

Pour cette dernière espèce, on a pu observer que certains animaux prélevaient de jeunes feuilles très appétissantes mais les rejetaient presque aussitôt.

Les vieilles feuilles de Lophira lanceolata et Piliostigma thonningii ne sont qu'en partie consommées.

Les bourgeons et feuilles au débourrement de Lannea schimperi et Erythrina sigmoidea, les jeunes feuilles et rameaux de Piliostigma thonningii et la quasi totalité des folioles de Daniellia oliveri sont consommés à l'exclusion des autres parties de ces plantes.

On note chez certains animaux une répugnance à consommer les feuilles d'arbres; ils ne s'y résignent que lorsqu'il n'y a vraiment plus rien à manger, donc parfois trop tard. Or il est important, à cause de leur haute valeur nutritive, que les feuilles des repousses successives soient consommées jeunes: il est donc bon de forcer les animaux, qui n'y sont pas habitués, à consommer des feuilles d'arbres avant qu'ils n'aient par trop maigri, donc aussitôt que possible dans la saison sèche. On y parvient en distribuant des rameaux à l'étable ou en surchargeant momentanément un parc fortement embuissonné.

\section{b) Analyses}

Les analyses sont repérées par un numéro d'ordre alphabétique et un nombre indiquant le mois et le millésime de prélèvement et un numéro d'ordre, si le même jour il y a eu pour la même espèce plusieurs prélèvements.
Les premiers prélèvements ont été effectués en février 1966 sur les parties des 5 espèces les plus appétées Daniellia oliveri (3-2-66), Hymenocardia acida (5-2-66), Lophira lanceolata (3-2-66), Piliostigma thonningii (3-2-66), Vitex madiensis (5-2-66).

En février 1967, la même opération a été pratiquée sur 6 espèces; on l'a accompagnée de mesures de rendement. Piliostigma thonningii (15-2-67),Daniellia oliveri (15-2-67), Gardenia ternifolia (27-2-67-1) (27-2-67-2), Hymenocardia acida (16-2-67), Lophira lanceolata (15-2-67), Vitex madiensis (16-2-67). En 1967, on détermina la composition et la valeur bromatologique d'une herbe de prairie à Brachiaria brizantha (17-2-67). Enfin en janvier 1968, les mesures de productivité et les prélèvements furent effectués seulement sur les feuilles de Daniellia oliveri à différents états de maturité; (analyses 10-1-68-1 - 10-1-68-2 - 10-1-68-3).

Des mesures furent faites également sur des repousses d'herbe de prairie sur pied; analyses (fin 1-68-1) (fin 1-68-2).

Le calcul de la valeur bromatologique posait évidemment le problème fondamental du choix des coefficients de digestibilité.

Pour les espèces ligneuses, en s'inspirant des chiffres cités dans l'ouvrage de PICCIONI (10) pour des espèces ligneuses européennes et méditerranéennes et de ceux donnés dans les tables de SCHNEIDER (14), on a retenu pour les plantes à faibles teneurs en cellulose les coefficients de digestibilité suivants :

$\begin{array}{lll}\text { Matières protéiques } & 60 & \text { p. } 100 \\ \text { Cellulose } & 60 & \text { p. } 100 \\ \text { Matières grasses } & 50 & \text { p. } 100 \\ \text { Extractif non azoté } & 75 & \text { p. } 100\end{array}$

... et pour les plantes à teneurs normales en cellulose respectivement 50 p. 100,50 p. 100 , 40 p. 100 et 60 p. 100.

Ces résultats sont certainement moins bons qu'en réalité; de cette façon les estimations seront faites par défaut, ce qui est préférable.

Pour les 4 graminées, les résultats ont été calculés à partir des tables hollandaises (5).

\section{ANALYSE DES RESULTATS}

Les résultats des analyses sont regroupés dans le tableau I; on y a adjoint quelques résul- 
TABLEAU $N^{\circ} \mathrm{I}$

Analyses bromatologiques de feullles d'arbres et de pousses d'herbes de saison sèche.

\begin{tabular}{|c|c|c|c|c|c|c|c|c|c|c|c|c|c|c|c|c|c|}
\hline $\begin{array}{c}\mathrm{N}^{\circ} \\
\text { Analyses }\end{array}$ & $\begin{array}{l}\text { Rêfêrences } \\
\text { êchantillons }\end{array}$ & $\begin{array}{l}\mathrm{H}_{2} \mathrm{O} \\
\mathrm{g} \cdot \\
\mathrm{p} \cdot \mathrm{I} 00\end{array}$ & $\begin{array}{c}\text { M. } \\
\text { sèches } \\
\text { p.100 }\end{array}$ & $\begin{array}{l}\text { M.P. } \\
\text { brutes } \\
\text { p.100 }\end{array}$ & $\begin{array}{c}\text { C. } \\
\text { g. } \\
\text { p. } 100\end{array}$ & $\begin{array}{c}\text { E. } \\
\text { Ththere } \\
\text { g. } \\
\text { p. } 100\end{array}$ & $\begin{array}{l}\text { Fan.A. } \\
\text { g. } \\
\text { p.100 }\end{array}$ & $\begin{array}{l}\text { M.i.'? } \\
\text { g. } \\
\text { p. } 100\end{array}$ & $\begin{array}{c}\text { T.C. } \\
\text { g. } \\
\text { p. } 100\end{array}$ & $\begin{array}{l}\mathrm{Ca} \\
\mathrm{g} \cdot \\
\mathrm{p} .100\end{array}$ & $\begin{array}{c}\mathrm{P} \\
\mathrm{g} . \\
\mathrm{p} .100\end{array}$ & $\begin{array}{c}\mathrm{Mg} \\
\mathrm{g} \cdot \\
\mathrm{p} .100\end{array}$ & $\begin{array}{l}\text { K. } \\
\text { g. } \\
\text { p. } 100\end{array}$ & I.f. & $\mathrm{UF} / \mathrm{k}_{\varepsilon}$ & $\begin{array}{l}\text { MPD } \\
\text { g. } \\
\text { p. } 100\end{array}$ & $\frac{\text { MPD }}{U F}$ \\
\hline 2722 & $\begin{array}{l}\text { Darielling otiveri } \\
\mathrm{N}^{\circ} \text { G4 } 3.2 .66 \\
\text { Jitunes reuilles }\end{array}$ & 78,60 & $\begin{array}{r}21,40 \\
160,00\end{array}$ & $\begin{array}{r}3,58 \\
16,74\end{array}$ & $\begin{array}{r}3,65 \\
17,05\end{array}$ & $\begin{array}{l}0,64 \\
2,98\end{array}$ & $\begin{array}{l}12,53 \\
58,57\end{array}$ & $\begin{array}{l}1,00 \\
4,66\end{array}$ & $\begin{array}{l}0,09 \\
0,44\end{array}$ & $\begin{array}{l}0,064 \\
0,300\end{array}$ & $\begin{array}{l}0,067 \\
0,313\end{array}$ & $\begin{array}{l}0,054 \\
0,254\end{array}$ & $\begin{array}{l}0,31 \\
1,47\end{array}$ & & $\begin{array}{l}0,17 \\
0,82\end{array}$ & $\begin{array}{l}2,15 \\
10,1\end{array}$ & 125 \\
\hline 3932 & $\begin{array}{c}\text { Daniellia oliveri } \\
15.2 .67 \\
\text { Jeunes feulles }\end{array}$ & 76,40 & $\begin{array}{r}23,60 \\
100,00\end{array}$ & $\begin{array}{r}3,46 \\
14,66\end{array}$ & $\begin{array}{r}5,35 \\
22,65\end{array}$ & $\begin{array}{l}0,80 \\
3,41\end{array}$ & $\begin{array}{l}1,2,84 \\
54,41\end{array}$ & $\begin{array}{l}1,15 \\
4,87\end{array}$ & $\begin{array}{l}0,11 \\
0,47\end{array}$ & $\begin{array}{l}0,127 \\
0,539\end{array}$ & $\begin{array}{l}0,056 \\
0,236\end{array}$ & $\begin{array}{l}0,068 \\
0,288\end{array}$ & $\begin{array}{l}0,32 \\
1,34\end{array}$ & $\begin{array}{l}12,20 \\
52,00\end{array}$ & $\begin{array}{l}0,18 \\
0,77\end{array}$ & $\begin{array}{l}2,17 \\
8,8\end{array}$ & 115 \\
\hline $484 I$ & $\begin{array}{l}\text { Daniettia oilueni } \\
\mathrm{N}^{\circ} 2249.1 .68 \\
\text { arbust. Ieuillus } \\
1 / 2 \text { mûres }\end{array}$ & 71.70 & $\begin{array}{r}28,30 \\
100,00\end{array} \mid$ & $\begin{array}{r}4,17 \\
14,73\end{array}$ & $\begin{array}{r}9,20 \\
32,60\end{array}$ & $\begin{array}{l}1,10 \\
3,90\end{array}$ & $\begin{array}{l}12,36 \\
43,57\end{array}$ & $\begin{array}{l}1,47 \\
5,20\end{array}$ & $\begin{array}{l}0,17 \\
0,60\end{array}$ & $\begin{array}{l}0,160 \\
0,566\end{array}$ & $\begin{array}{l}0,057 \\
0,202\end{array}$ & $\begin{array}{l}0,097 \\
0,342\end{array}$ & $\begin{array}{l}0,39 \\
1,39\end{array}$ & $\begin{array}{l}14,10 \\
50,80\end{array}$ & $\begin{array}{l}0,14 \\
0,5\end{array}$ & $\begin{array}{l}2,1 \\
7,5\end{array}$ & $I b 0$ \\
\hline 4842 & $\begin{array}{l}\text { Duniellics olive } x^{2} \\
\mathrm{~N}^{\circ} 22510.1 .68 \\
\text { arbres }\end{array}$ & 82,15 & $\begin{array}{l}17,85 \\
100,00\end{array}$ & $\begin{array}{r}4,10 \\
22,97\end{array}$ & $\begin{array}{r}4,35 \\
24,35\end{array}$ & $\begin{array}{l}1,08 \\
6,03\end{array}$ & $\begin{array}{r}7,18 \\
40,29\end{array}$ & $\begin{array}{l}1,14 \\
6,36\end{array}$ & $\begin{array}{l}0,07 \\
0,37\end{array}$ & $\begin{array}{l}0,070 \\
0,391\end{array}$ & $\begin{array}{l}0,076 \\
0,424\end{array}$ & $\begin{array}{l}0,065 \\
0,363\end{array}$ & $\begin{array}{l}0,41 \\
2,30\end{array}$ & $\begin{array}{r}6,80 \\
38,20\end{array}$ & $\begin{array}{l}0,13 \\
0,75\end{array}$ & $\begin{array}{l}2,46 \\
13,8\end{array}$ & 190 \\
\hline 4843 & 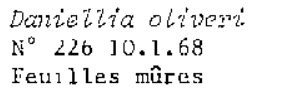 & 67,35 & $\begin{array}{r}32,65 \\
100,00\end{array}$ & $\begin{array}{r}5,72 \\
17,52\end{array}$ & $\begin{array}{l}10,10 \\
31,00\end{array}$ & $\begin{array}{l}1,19 \\
3,65\end{array}$ & $\begin{array}{l}13,76 \\
42,07\end{array}$ & $\begin{array}{l}1,88 \\
5,76\end{array}$ & $\begin{array}{l}0,30 \\
0,93\end{array}$ & $\begin{array}{l}0,240 \\
0,735\end{array}$ & $\left\{\begin{array}{l}0,072 \\
0,220\end{array}\right.$ & $\begin{array}{l}0,117 \\
0,359\end{array}$ & $\begin{array}{l}0,37 \\
1,13\end{array}$ & $\begin{array}{l}16,80 \\
51,70\end{array}$ & $\begin{array}{l}0,65 \\
0,5\end{array}$ & $\begin{array}{l}2,86 \\
8,75\end{array}$ & 175 \\
\hline 3929 & $\begin{array}{l}\text { Gardoniut termijoila } \\
\qquad 27,2.67 \\
\text { Heullles dusstichés } \\
\text { apris ieu }\end{array}$ & 6,65 & $\begin{array}{r}93,35 \\
100,00\end{array}$ & $\begin{array}{l}10,43 \\
11,17\end{array}$ & $\begin{array}{l}17,90 \\
19,20\end{array}$ & $\begin{array}{l}0,70 \\
0,75\end{array}$ & $\begin{array}{l}57,15 \\
61,20\end{array}$ & $\begin{array}{l}7,17 \\
7,68\end{array}$ & $\begin{array}{l}0,17 \\
0,18\end{array}$ & $\begin{array}{l}0,349 \\
0,910\end{array}$ & $\left\{\begin{array}{l}0,327 \\
0,350\end{array}\right.$ & $\begin{array}{l}0,422 \\
0,452\end{array}$ & $\begin{array}{l}2,12 \\
2,27\end{array}$ & $\begin{array}{l}31,30 \\
33,60\end{array}$ & $\begin{array}{l}0,68 \\
0,73\end{array}$ & $\begin{array}{l}6,25 \\
6,7\end{array}$ & 90 \\
\hline 3930 & $\begin{array}{c}\text { Gardenia ternifolza } \\
\qquad 2,2.67\end{array}$ & 60,60 & $\begin{array}{r}39,40 \\
100,00\end{array}$ & $\begin{array}{l}3,35 \\
8,49\end{array}$ & $\begin{array}{r}8,30 \\
21,05\end{array}$ & $\begin{array}{l}0,96 \\
2,45\end{array}$ & $\begin{array}{l}22,99 \\
58,35\end{array}$ & $\begin{array}{l}3,80 \\
9,66\end{array}$ & $\begin{array}{l}0,07 \\
0,17\end{array}$ & $\begin{array}{l}0,667 \\
1,694\end{array}$ & $\mid \begin{array}{l}0,049 \\
0,125\end{array}$ & $\begin{array}{l}0,200 \\
0,508\end{array}$ & $\begin{array}{l}1,00 \\
2,53\end{array}$ & $\begin{array}{l}14,30 \\
36,40\end{array}$ & $\begin{array}{l}0,26 \\
0,66\end{array}$ & $\begin{array}{l}2 \\
5,1\end{array}$ & 80 \\
\hline 2719 & $\begin{array}{c}\text { Himenocardia acida } \\
5.2 .66\end{array}$ & 76,10 & $\begin{array}{r}23,90 \\
100,00\end{array}$ & $\begin{array}{l}2,25 \\
9,41\end{array}$ & $\begin{array}{r}6,90 \\
28,85\end{array}$ & $\begin{array}{l}1,38 \\
5,79\end{array}$ & $\begin{array}{l}10,90 \\
45,63\end{array}$ & $\begin{array}{r}2,47 \\
10,32\end{array}$ & $\begin{array}{l}0,66 \\
2,77\end{array}$ & $\begin{array}{l}0,091 \\
0,382\end{array}$ & $\begin{array}{l}0,068 \\
0,285\end{array}$ & $\begin{array}{l}0,042 \\
0,177\end{array}$ & $\begin{array}{l}0,90 \\
3,75\end{array}$ & & $\begin{array}{l}0,11 \\
0,47\end{array}$ & $\begin{array}{l}1,12 \\
4,7\end{array}$ & 100 \\
\hline 3931 & $\begin{array}{c}\text { Hymenocurdia achla } \\
16.2 .67\end{array}$ & 80,35 & $\begin{array}{r}19,65 \\
100,00\end{array} \mid$ & $\begin{array}{r}2,31 \\
11,75\end{array}$ & $\begin{array}{r}4,45 \\
32,75\end{array}$ & $\begin{array}{l}1,13 \\
5,75\end{array}$ & $\begin{array}{l}11,07 \\
56,22\end{array}$ & $\begin{array}{l}0,69 \\
3,53\end{array}$ & $\begin{array}{l}0,04 \\
0,22\end{array}$ & $\begin{array}{l}0,066 \\
0,338\end{array}$ & $\begin{array}{l}0,042 \\
0,216\end{array}$ & $\begin{array}{l}0,062 \\
0,316\end{array}$ & $\begin{array}{l}0,18 \\
0,92\end{array}$ & $\begin{array}{r}8,80 \\
44,90\end{array}$ & $\begin{array}{l}0,11 \\
0,56\end{array}$ & $\begin{array}{l}1,15 \\
5,85\end{array}$ & 105 \\
\hline 2721 & 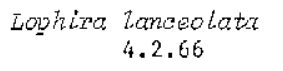 & 76,80 & $\begin{array}{r}23,20 \\
100,00\end{array}$ & $\begin{array}{r}3,41 \\
14,69\end{array}$ & $\begin{array}{r}3,00 \\
12,95\end{array}$ & $\begin{array}{l}0,42 \\
1,43\end{array}$ & $\begin{array}{l}15,83 \\
68,19\end{array}$ & $\begin{array}{l}0,54 \\
2,34\end{array}$ & $\begin{array}{l}0,05 \\
0,22\end{array}$ & $\begin{array}{l}0,035 \\
0,152\end{array}$ & $\left\{\begin{array}{l}0,058 \\
0,252\end{array}\right.$ & $\begin{array}{l}0,011 \\
0,046\end{array}$ & $\begin{array}{l}0,16 \\
0,67\end{array}$ & & $\begin{array}{l}0,19 \\
0,83\end{array}$ & $\begin{array}{l}2,05 \\
8,8,\end{array}$ & 105 \\
\hline
\end{tabular}




\begin{tabular}{|c|c|c|c|c|c|c|c|c|c|c|c|c|c|c|c|c|c|}
\hline \multirow{2}{*}{$\begin{array}{c}\varsigma \varepsilon \\
\ldots \\
\varsigma \xi\end{array}$} & \multirow{2}{*}{$z^{6} z$} & \multirow{2}{*}{$29^{\circ} 0$} & \multicolumn{15}{|c|}{ 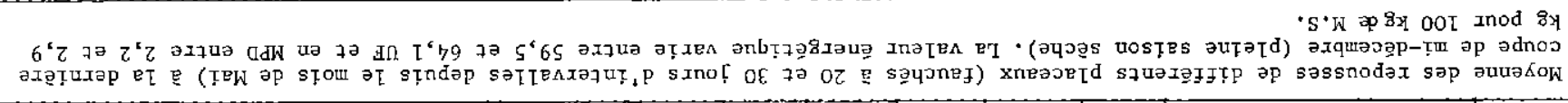 } \\
\hline & & & $\begin{array}{l}\varsigma L^{*} 6 \varepsilon \\
\varsigma \gg * 8 \mathrm{~L}\end{array}$ & $\begin{array}{l}\angle L{ }^{\circ} 0 \\
9 E^{\circ} 0\end{array}$ & $\begin{array}{l}\text { Ioz'o } \\
\varepsilon 60^{\circ} 0\end{array}$ & $\begin{array}{l}\angle 8 \mathrm{I}^{\circ} \circ \\
\angle 80^{\circ} \circ\end{array}$ & $\begin{array}{l}819^{\circ} 0 \\
\angle 82^{\circ} 0\end{array}$ & $\begin{array}{l}98 \times 8 \\
Z I \times 7\end{array}$ & $\begin{array}{l}8 Z^{\prime} z \mathrm{Z} \\
0 L \subset \mathrm{S}\end{array}$ & $\begin{array}{l}86 \times 87 \\
7 L^{\prime} z z\end{array}$ & $\begin{array}{l}L 7^{\circ} \mathrm{L} \\
59^{\circ} 0\end{array}$ & $\begin{array}{l}O 8^{\prime} \varepsilon \varepsilon \\
O L^{\prime} \subseteq \mathrm{L}\end{array}$ & $\begin{array}{l}8 \mathcal{S}^{\mathrm{c}} \varepsilon \\
99^{6} \mathrm{~T}\end{array}$ & $\begin{array}{l}00^{\circ} 00 \mathrm{~T} \\
5 \rightarrow{ }^{\circ} 97\end{array}$ & $\varsigma \varsigma^{\prime} \varepsilon \varsigma$ & 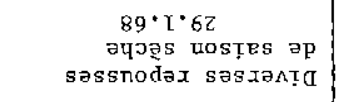 & 0067 \\
\hline$\varsigma 9$ & $7^{\circ} \varepsilon$ & $Z 5^{\circ} 0$ & $\begin{array}{l}08^{\circ} \mathrm{IZ} \\
S E^{\circ} \mathrm{ZZ}\end{array}$ & $\begin{array}{l}\mathrm{I} z^{*} \mathrm{I} \\
\mathrm{s} 9^{\star 0}\end{array}$ & $\begin{array}{l}\angle O Z \div O \\
\text { OLI:O }\end{array}$ & $\begin{array}{l}Y \angle I \times 0 \\
L 60 * 0\end{array}$ & $\begin{array}{l}\tau z+7^{\circ} 0 \\
5 z Z^{\circ} 0\end{array}$ & $\begin{array}{l}O I^{\prime} \cdot 9 \\
9 Z^{\circ} \varepsilon\end{array}$ & $\begin{array}{l}88 * 6 \\
9 Z^{\circ} 5\end{array}$ & $\begin{array}{l}60^{\circ} 97 \\
\angle 9^{\circ}+2 z\end{array}$ & $\begin{array}{l}66^{\mathrm{t}} \mathrm{T} \\
90^{\circ} \mathrm{T}\end{array}$ & $\begin{array}{l}\subsetneq Z^{\prime} \varsigma \varepsilon \\
58 \times 8 \mathrm{I}\end{array}$ & $\begin{array}{l}78^{\circ} 9 \\
99^{\circ} \varepsilon\end{array}$ & $\begin{array}{l}00^{\circ} 00 \mathrm{~L} \\
05^{c} E S\end{array}$ & $\cos 97$ & 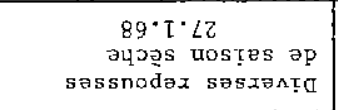 & 068 म \\
\hline$E I$ & $\varsigma$ & $98 \varepsilon^{\circ} 0$ & $\begin{array}{l}O S^{4} 97 \\
O L^{*} \mathrm{IE}\end{array}$ & $\begin{array}{l}59^{\circ} 0 \\
b t^{\circ} 0\end{array}$ & $\begin{array}{l}\varepsilon O z^{*} \circ \\
6 E I * 0\end{array}$ & $\begin{array}{l}\mathrm{T} 9 \tau^{\circ} \circ \\
8 L T \cdot 0\end{array}$ & $\begin{array}{l}9+77^{\circ} 0 \\
\text { SOE } 0\end{array}$ & $\begin{array}{l}\tau \varepsilon^{*} 9 \\
T \varepsilon^{*}+{ }^{\prime}\end{array}$ & $\begin{array}{l}\text { of' } 6 \\
98 \times 9\end{array}$ & $\begin{array}{l}\angle \varepsilon^{\prime} \angle t \\
S \varepsilon^{\circ} Z \varepsilon\end{array}$ & $\begin{array}{l}60^{\circ} \mathrm{T} \\
S L^{\circ} \mathrm{O}\end{array}$ & $S L^{\prime} 6 \varepsilon$ & $\begin{array}{l}75^{\circ} ? \\
7 L^{*} I\end{array}$ & $\begin{array}{l}00<00 T \\
5 \varepsilon \times 89\end{array}$ & $59^{\prime} T E$ & 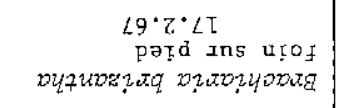 & 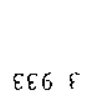 \\
\hline 56 & $\begin{array}{l}\mathrm{SI} " \mathrm{~L} \\
\mathrm{~S} \cdot \mathrm{T}\end{array}$ & $\begin{array}{l}5 L^{\circ} \circ \\
9 I^{\circ} 0\end{array}$ & $\begin{array}{l}00^{\prime} 8 \varepsilon \\
06^{\circ} L\end{array}$ & $\begin{array}{l}29^{\circ} z \\
\varsigma \varsigma^{\circ} 0\end{array}$ & $\begin{array}{l}\varepsilon \varepsilon 7^{\circ} \circ \\
\tau 60^{\circ} \circ\end{array}$ & $\begin{array}{l}6.6 L^{\circ} 0 \\
1700^{\circ} 0\end{array}$ & $\begin{array}{l}867^{\circ} 0 \\
\text { SOI'0 }\end{array}$ & $\begin{array}{l}82^{\circ} 0 \\
70 \circ 0\end{array}$ & $\begin{array}{l}\angle I^{\prime} \angle \\
05^{\prime} L\end{array}$ & $\begin{array}{l}70 * \angle 5 \\
66^{\circ} \mathrm{LI}\end{array}$ & $\begin{array}{l}70^{\circ} \varepsilon \\
79^{\circ}\end{array}$ & $\begin{array}{l}00^{c} \mathrm{IZ} \\
0+7 \mathrm{t}\end{array}$ & $\begin{array}{l}S L^{c} \mathrm{IT} \\
\angle H^{\circ} \mathrm{Z}\end{array}$ & $\begin{array}{l}00^{\circ} 00 \mathrm{t} \\
00^{\circ} \mathrm{tz}\end{array}$ & $00^{\circ} 6 L$ & $\begin{array}{l}\angle 9^{\circ} \tau \cdot 9 \mathrm{~L} \\
\text { s.suozpou } x 27 . \Lambda\end{array}$ & $\angle Z 6 \&$ \\
\hline OTt & $\begin{array}{l}5^{\prime} 8 \\
8 ، T\end{array}$ & $\begin{array}{l}6 L^{\circ} 0 \\
\angle I{ }^{\circ} 0\end{array}$ & & $\begin{array}{l}\mathrm{L} L^{\prime} \mathrm{Z} \\
6 \mathrm{I}^{\prime} \mathrm{O}\end{array}$ & $\begin{array}{l}\text { EगI‘0 } \\
\text { OTO‘0 }\end{array}$ & $\begin{array}{l}982^{\circ} \circ \\
020 \circ \circ\end{array}$ & $\begin{array}{l}897^{\circ} 0 \\
2 \varepsilon 0^{\circ} 0\end{array}$ & $\begin{array}{l}9 I^{\circ} 0 \\
T 0^{\circ} 0\end{array}$ & $\begin{array}{l}7 z^{6} \angle \\
95^{\circ} \mathrm{I}\end{array}$ & $\begin{array}{r}z Z^{\prime} \angle S \\
9^{\circ} Z \mathrm{I}\end{array}$ & $\begin{array}{l}\angle L^{\circ} \varepsilon \\
18^{*} 0\end{array}$ & $\begin{array}{l}O L^{\prime} \angle T \\
S L^{\prime} E\end{array}$ & $\begin{array}{c}\angle 0^{c} \mapsto T \\
\varepsilon\end{array}$ & $\begin{array}{c}\infty^{\prime} 00 \mathrm{~L} \\
I^{\prime} \mathrm{T} z\end{array}$ & 6.81 & $\begin{array}{l}99^{\circ} \mathrm{Z} \cdot \mathrm{s} \\
\text { 8.suว?pou waqu }\end{array}$ & $0 \pi \angle Z$ \\
\hline Ol I & $\begin{array}{l}9 \times 8 \\
6 * 1\end{array}$ & $\begin{array}{l}8 L^{\circ} 0 \\
\angle T^{\circ} 0\end{array}$ & $\begin{array}{l}05^{\circ} 07 \\
00^{\circ} 6\end{array}$ & $\begin{array}{l}S S^{\circ} \tau \\
7 \varepsilon^{\circ} 0\end{array}$ & $\begin{array}{l}\angle L I \% O \\
6 E O^{\circ} 0\end{array}$ & 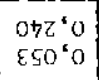 & $\begin{array}{l}897^{\circ} \circ \\
70 c^{\circ} 0\end{array}$ & $\begin{array}{l}2 T^{\circ} 0 \\
80 \% 0\end{array}$ & $\begin{array}{l}28 \times \mapsto \\
\angle O^{*} \mathrm{I}\end{array}$ & $\begin{array}{l}z z^{*} 65 \\
8 I * c \tau\end{array}$ & $\begin{array}{l}\eta Z^{c} \varepsilon \\
Z L^{\circ} \circ\end{array}$ & 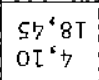 & $\begin{array}{l}\angle Z^{*} \gg \mathrm{L} \\
8 \mathrm{I} \times\end{array}$ & $\begin{array}{l}00^{\prime} \mathrm{OOI} \\
s \mathrm{C}^{\circ} \mathrm{Zz}\end{array}$ & $s L^{\prime} \angle L$ & 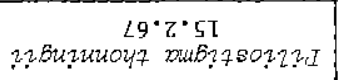 & $756 \varepsilon$ \\
\hline OEI & $\begin{array}{l}\text { ot } \\
z\end{array}$ & $\begin{array}{l}\angle L C^{\circ} \\
S I \\
S C_{0}\end{array}$ & & $\begin{array}{l}20 \% 2 \\
0+0\end{array}$ & $\begin{array}{l}01 z^{*} \circ \\
\pm \neq 0 \div 0\end{array}$ & $\begin{array}{l}65 \varepsilon^{\circ} 0 \\
T \angle 0^{\circ} \circ\end{array}$ & $\begin{array}{l}818^{\circ} \circ \\
890^{\circ} \circ\end{array}$ & $\begin{array}{l}\varepsilon Z^{*} \circ \\
S 0^{\circ} \circ\end{array}$ & $\begin{array}{l}8 Z^{\circ} 5 \\
70 \div 5\end{array}$ & $\begin{array}{l}97^{\circ} 6 \leftrightarrows \\
\varepsilon L^{5}[L\end{array}$ & $\begin{array}{l}\varsigma 8^{\circ} Z \\
9 \varsigma^{\circ} 0\end{array}$ & $\begin{array}{l}\varsigma \varsigma^{\prime} \sqsubseteq \mathrm{I} \\
\varsigma 0^{\circ} \mathrm{E}\end{array}$ & $\begin{array}{l}98^{\circ} 9 \mathrm{~L} \\
Z E^{\prime} \mathrm{E}\end{array}$ & $\begin{array}{l}00^{\circ} 00 \mathrm{~T} \\
0 L^{\circ} 6 \mathrm{~T}\end{array}$ & of'08 & 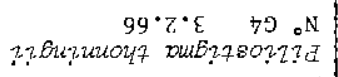 & $\varepsilon Z L 乙$ \\
\hline sol & $\begin{array}{l}9^{6} s \\
S^{*}[\end{array}$ & $\begin{array}{l}75^{\circ} \circ \\
5 \mathrm{\circ}: 0\end{array}$ & $\begin{array}{l}08^{\circ} 05 \\
0 g^{\prime} \mathrm{CI}\end{array}$ & $\begin{array}{l}I L^{c} O \\
6 I^{\prime} 0\end{array}$ & $\begin{array}{l}E \geqslant \tau \div 0 \\
6 \varepsilon 0<0\end{array}$ & $\begin{array}{l}z 7 \tau^{\circ} 0 \\
8 \varepsilon 0^{\circ} 0\end{array}$ & $\begin{array}{l}2 \nmid 0^{\circ} 0 \\
8 E 0 \div 0\end{array}$ & $\begin{array}{l}60 \% 0 \\
20 \% 0\end{array}$ & $\begin{array}{l}277^{\circ} 0 \\
59^{\circ} 0\end{array}$ & $\begin{array}{l}z \varepsilon^{\prime} 8 \varsigma \\
\xi L “ \varsigma L\end{array}$ & $\begin{array}{l}\angle 6^{\circ} \tau \\
\varepsilon \varsigma^{\circ} 0\end{array}$ & $\begin{array}{l}\text { Oz' } 9 Z \\
50 \times L\end{array}$ & $\begin{array}{l}0 \mathrm{I}^{\circ} \mathrm{TI} \\
66^{\circ} \mathrm{z}\end{array}$ & $\begin{array}{l}00^{\circ} 00 \tau \\
56^{\circ} 9 z\end{array}$ & $50^{\circ} \varepsilon L$ & 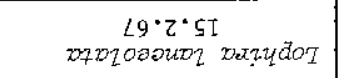 & ऽE $6 \&$ \\
\hline
\end{tabular}

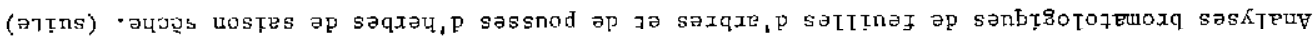

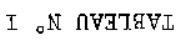


tats d'analyses de fourrages de graminées, disponibles en saison sèche sans feux, dans les conditions pastorales de l'Adamaoua.

1. Comme pour les espèces européennes (10), la teneur en matières protéiques brutes est remarquablement élevée surtout pour la saison considérée et par rapport aux autres aliments naturellement disponibles. Elle est par exemple pour Daniellia oliveri de 14,5 à $23 \mathrm{~g}$ p. $100 \mathrm{~g}$ de matière sèche; pour Piliostigma thonningii de 14 à $17 \mathrm{~g} \mathrm{p}$. $100 \mathrm{~g}$ de M.S. et pour les autres espèces elle atteint rarement moins de 10 p. 100 , alors que pour la grosse masse de fourrage sur pied appétée à la même époque, elle se situe au-dessous de 5 p. 100. C'est ce qui fait l'intérêt majeur de cette source alimentaire.

2. La valeur énergétique est toujours assez élevée pour satisfaire théoriquement aux besoins d'entretien du bétail et même de crôt (200 g/jour pour un animal de $250 \mathrm{~kg}$ ), compte tenu des possibilités qu'ont ces animaux d'ingérer de la matière sèche $(2,5 \mathrm{~kg} \mathrm{p} .100 \mathrm{~kg}$ de poids vif).

3. Les besoins d'entretien d'un animal de $250 \mathrm{~kg}$ en calcium et phosphore sont estimés respectivement à $12,5 \mathrm{~g}$ et $7,5 \mathrm{~g}$ avec en outre un rapport $\frac{\mathrm{P}}{\mathrm{Ca}}$ variant entre 0,3 et 1,2 (4).

A ce point de vue :

- Lophira lanceolata est trop pauvre en calcium;
- Gardenia ternifolia frais est pauvre en phosphore;

- Gardenia ternifolia a un rapport $\frac{\mathrm{P}}{\mathrm{Ca}}$ beaucoup trop faible, et

- Lophira lanceolata (2721) un rapport $\frac{\mathrm{P}}{\mathrm{Ca}}$ trop élevé.

4. Quant aux teneurs en potassium (10 g au maximum pour $100 \mathrm{~kg}$ vifs), correctes pour le Lophira, elles apparaissent comme excessives pour toutes les autres espèces, en particulier pour Vitex madiensis et Hymenocardia acida. Ces fortes teneurs peuvent être acceptées si une complémentation en chlorure de sodium permet de conserver le rapport $\frac{\mathrm{K}}{\mathrm{Na}}$ inférieur à 4 .

5. Concernant le magnésium LEROY (8) indique que le rapport $\frac{\mathrm{Ca}}{\mathrm{Mg}}$ doit se maintenir entre 3 et 4 et WIND (6) que le rapport $\frac{\mathrm{K}}{\mathrm{Ca}+\mathrm{Mg}}$ qui mesure en quelque sorte le risque de tétanie de nutrition doit être inférieur à 2,37 en milliéquivalents.

A ce point de vue les teneurs en magnésium sont faibles et les rapports indiqués ci-dessus n'ont que rarement pour les différentes espèces une valeur acceptable, comme l'indique le tableau 2 suivant où il apparaît que Hymenocardia acida est le plus déséquilibré.

\begin{tabular}{|c|c|c|}
\hline & $\frac{\mathrm{Ca}}{\mathrm{Mq}}$ & $\frac{k}{(\mathrm{Ca}+\mathrm{Mq}) \mathrm{mEq}}$ \\
\hline Valeur optima & 3 ì 4 & inf., à 2,37 \\
\hline Danieztia oliveri & 1,1 à 2 & 0,45 à 1,2 \\
\hline Pitiostigma thonningit & 1,5 et 2,65 & 1,57 et 1,07 \\
\hline Gardenia temifotia & 2 et 3,3 & 0,71 et 0,52 \\
\hline Lophira Zanceotata & 3,3 et 1 & 1,56 et 1 \\
\hline Iymenocardia acida & 2,18 et 1,07 & 2,9 et 0,55 \\
\hline Vitex madiensis & 3,28 et 1,15 & et 1,15 \\
\hline
\end{tabular}

Qucls que soient les déficits minéraux du pâturage arboré, il faut bien considérer qu“il apporte un appoint substantiel en protéines alors que le pâturage herbacé en est pratiquement dépourvu et que des compensations minérales sont possibles avec ce dernier. 


\begin{tabular}{|c|c|c|c|c|c|c|c|c|}
\hline $\begin{array}{l}\stackrel{+}{0} \\
\stackrel{+}{0} \\
\stackrel{0}{E}\end{array}$ & 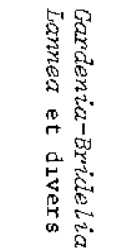 & 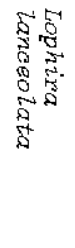 & 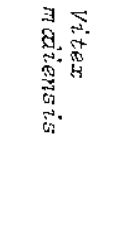 & 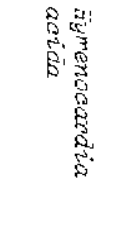 & 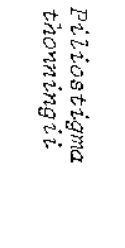 & 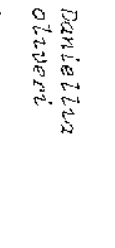 & & $\begin{array}{l}m \\
n \\
0 \\
0 \\
n \\
n \\
n\end{array}$ \\
\hline 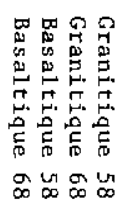 & 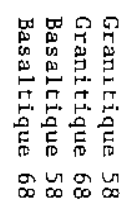 & 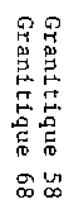 & 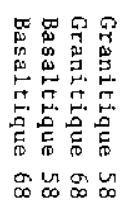 & 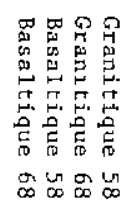 & 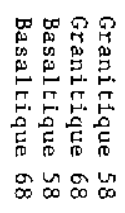 & 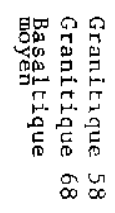 & 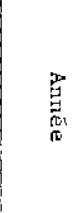 & 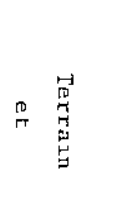 \\
\hline $\begin{array}{l}\omega \\
\omega \\
\omega \\
\omega\end{array}$ & $\infty N \stackrel{\omega}{\infty}$ & $\begin{array}{l}\text { 占怘 } \\
\text { in }\end{array}$ & Nat a & $\underset{\sigma}{\sim} \stackrel{\sim}{N} D_{\infty}^{\circ}$ & 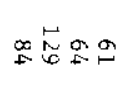 & N & $\underset{\substack{0 \\
0 \\
0 \\
0 \\
0}}{0}$ & \\
\hline 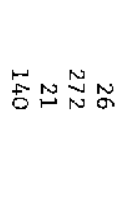 & w & $\begin{array}{l}\text { No } \\
\text { in }\end{array}$ & 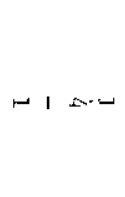 & 㭗 & $\vec{\sigma} \approx \omega$ & $i_{\omega}^{\infty} \stackrel{\infty}{\infty}$ & 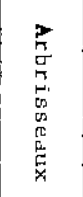 & 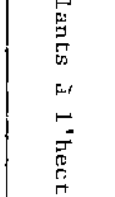 \\
\hline 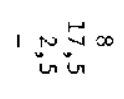 & 1 1 N & in & $\begin{array}{llll}1 & 1 & 1 & 1\end{array}$ & $10 \times 1$ & $1 * 11$ & $\begin{array}{l}N \text { on } \\
\text { in in }\end{array}$ & 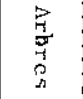 & \\
\hline$\underset{N}{\infty} \stackrel{心}{心}$ & 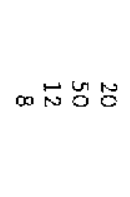 & 它出 & $A N \sigma^{\omega}$ & 낭 용 & 봉ํํำ & 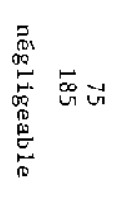 & 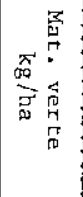 & 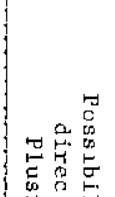 \\
\hline 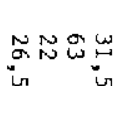 & H & viv & N & 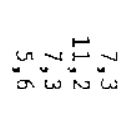 & 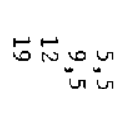 & $\stackrel{\omega}{o}$ & 家 & 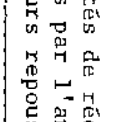 \\
\hline من & 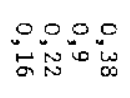 & 里 & $\begin{array}{l}0.00 \\
0000 \\
0.00\end{array}$ & 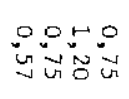 & N口50 & $\begin{array}{l} \pm 5 \\
\operatorname{Ln}^{\infty}\end{array}$ & 愛杲 & 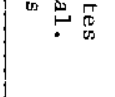 \\
\hline 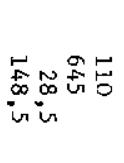 &,$\quad$, g & जे 1 & 夏 & 8,5, & 象苍峞 & $\infty \stackrel{t}{0} \stackrel{0}{0}$ & 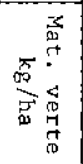 & 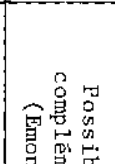 \\
\hline 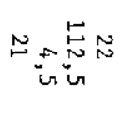 & $11 \underbrace{\infty}{ }^{\infty}$ & $\infty 1$ & 苜 & $\begin{array}{ll}0 . & 151 \\
\text { in } & \text { N }\end{array}$ & 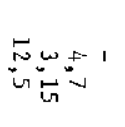 & 岕肖怘 & $\frac{\text { 孚 }}{\stackrel{p}{p}}$ & 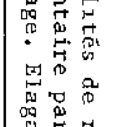 \\
\hline 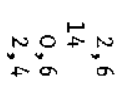 & 110 & 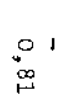 & $\dot{9}$ & 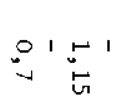 & $\begin{array}{l}-001 \\
4=0\end{array}$ & 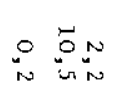 & 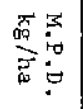 & 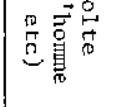 \\
\hline
\end{tabular}




\section{RENDEMENTS QUANTITATIFS}

Utilisant les inventaires signalés dans le précédent article (13) une estimation des quantités de feuilles exploitables en saison sèche par le bétail a été faite.

Aux 6 espèces signalées précédemment on a ajouté celles qui sont habituellement bien appétées et qui sont abondantes Bridellia Spp. Lannea schimperi, Cussonia barteri, etc.

Ainsi le tableau 3 donne d'une part les possibilités de récolte directe par les animaux tout au long de la saison sèche et d'autre part les possibilités de récolte par l'homme utilisant soit l'élagage, soit l'émondage, soit l'abaissement des branches qui les rend accessibles aux animaux sans les détacher du tronc.

C'est de loin Daniellia oliveri qui présente le plus d'intérêt en terrain granitique et il se trouve heureusement que c'est aussi l'espèce la mieux appétée. Arbre de savane de première grandeur, il offre des possibilités d'émondage extrêmement intéressantes, 2 à 3 dans la saison; des jeunes feuilles apparaissent relativement tôt après broutage ou émondage ce qui donne à l'arbre une allure de fraîcheur exceptionnelle en raison des tons rouges infiniment variés qu'elles présentent au débourrement qui est relativement groupé dans le temps pour la grosse majorité des sujets.

Piliostigma thonningii très intéressant en terrains basaltiques débourre nettement plus tardivement en moyenne que le Daniellia oliveri, mais pâturé, il donne l'impression de fournir des feuilles «en continu» que les animaux viennent grapiller.

Quant à Hymenocardia acida, ses jeunes feuilles glutineuses sont facilement délaissées par le bétail non habitué à les consommer ou pas assez affamé. Or très rapidement, ces feuilles deviennent coriaces et ne sont pas consommées. Appété par le bétail, Hymenocardia acida se comporte comme Piliostigma thonningii; il est brouté tout au long de la saison sèche après la première pousse.

Lophira lanceolata a, lui aussi, très rapidement des feuilles trop dures pour être consommées et, très tôt, les animaux n'en prennent que l'extrémité. Comme pour Cussonia barteri, l'allure peu buissonnante des individus ne permet qu'une exploitation relativement faible par les animaux et donne tout son intérêt à l'intervention humaine.

Bridelia Spp, Lannea schimperi, Erythrina sigmoidea, etc... nécessitent eux aussi de par leur port un émondage pour que soit utilisé tout ce qu'ils peuvent donner.

En définitive, on voit la très nette supériorité des zones granitiques sur les basaltiques pour l'appoint arboré, mais cela essentiellement grâce à la présence de Daniellia oliveri qui cependant n'est pas dans tout l'Adamaoua aussi abondant que dans les parcs du centre de Wakwa. Par contre, certains secteurs basaltiques de cette région sont aussi riches en ligneux que les zones granitiques et leur pâture arborée doit y être aussi importante.

En résumé, la pâture arborée des différentęs espèces peut fournir suivant l'état d'embuissonnement du terrain :

1. A l'exploitation libre par les animaux de 150 à $500 \mathrm{~kg}$ de matière verte à l'hectare contenant 24 p. 100 de matière sèche, $21 \mathrm{~g}$ de matière protéique digestible (M.P.D.) par $\mathrm{kg}$ de matière verte valant 0,15 unité fourragère au $\mathrm{kg}$.

Le rapport $\frac{\mathrm{MPD}}{\mathrm{UF}}$ étant égal à 130 et le coefficient d'encombrement à 1,5 , cet aliment pourrait à lui seul assurer la ration d'un bovin de $250 \mathrm{~kg}$ prenant $500 \mathrm{~g} /$ jour ou d'une mère de $250 \mathrm{~kg}$ fournissant $4 \mathrm{~kg}$ de lait à 4 p. 100 de matière grasse, ce qui, en pleine saison sèche, serait un succès remarquable.

2. Avec une exploitation complémentaire par l'homme, on pourrait tirer au total, suivant les terrains, de 180 à $1.100 \mathrm{~kg}$ de matière verte ayant sensiblement la même valeur que celle indiquée ci-dessus.

\section{COMPORTEMENT DES TROUPEAUX}

\section{a) Sur parcours en terrains granitiques brâlés régulièrement}

Dans des essais dont les résultats ont été publiés en 1966 (11) il était précisé que, pour des parcs fortement embuissonnés dans lesquels on dénombrait à l'hectare 40 arbres, 900 arbrisseaux et 1.700 rejets, la pâture arborée d'un hectare permettrait d'entretenir pendant 4 jours un animal de $400 \mathrm{~kg}$, c'est-à-dire fournirait 17 UF et $1 \mathrm{~kg}$ de MPD (au minimum). 
TABLEAU N $N^{\circ}$ IV

\begin{tabular}{|c|c|c|c|c|c|c|}
\hline & Rejets & Arbustes & Arbres & M.V. & U. F. & M.P.D. \\
\hline & à I'Ha & à $l^{\prime} \mathrm{Ha}$ & à I'Ha & en $\mathrm{kg} / \mathrm{Ha}$ & à $1{ }^{\prime} \mathrm{Ha}$ & en $\mathrm{kg} / \mathrm{Ha}$ \\
\hline Danieziza oiviveri & 195 & 33 & 7,5 & 95 & 15 & 2 \\
\hline Ilymenoadrita acida & 140 & 122 & 0,5 & 130 & 14,5 & 1,5 \\
\hline Fitiostigma thonningit & 65 & 30 & 1 & 60 & 9,5 & 1,1 \\
\hline Viten madionsis & 15 & 3 & - & 10 & 1,6 & 0,16 \\
\hline Lophira Ianoeotata & 53 & 77 & 3 & 35 & 6,4 & 0,68 \\
\hline Divers & 82 & 37 & 3 & 80 & 14 & 1,52 \\
\hline Total & 550 & 302 & 15 & 410 & 61 & 6,96 \\
\hline
\end{tabular}

La composition de la formation ligneuse appétée des parcs utilisés est précisée au Tableau IV.

Ce tableau montre que les rations théoriquement récupérables sont de beaucoup supérieures à celles que nous avons alors estimées: $61 \mathrm{UF}$ au lieu de 17.

Cette différence tient au fait que nous n'avons pas tenu compte de la pâture arborée effectuée pendant les premiers jours de charge, estimant que la ration était alors exclusivement fournie par le regain herbacé. Or, en fait, nos récentes observations nous ont montré que le $1 / 3$ de la ration était d'origine arborée, ce qui double la productivité estimée 34 UF $2 \mathrm{~kg}$ de M.P.D. au lieu de 17 et 2 respectivement.

Le reste de la différence tient essentiellement au fait que le passage des animaux dans les parcs fut très bref, et qu'entre 2 passages, les éléments pâturables étaient devenus inconsommables. En particulier, les Hymenocardia acida ne furent à peu près pas touchés.

\section{b) Sur terrain mixte basaltique rouge, latéritique, granitique}

Un troupeau de 21 vaches de race Foulbé a occupé pendant la saison des pluies un parc de 30 ha avec une charge de $250 \mathrm{~kg} / \mathrm{ha}$; il est resté sur ce parc pendant la saison sèche.

Les refus y étaient nombreux, constitués essentiellement par les touffes de $80 \mathrm{~cm}$ à $1 \mathrm{~m}$ de haut des grandes andropogonées cespiteuses (Hyparrhenia Spp, Andropogon gayanus, etc...) et de Panicum phragmitoides.

Dès la fin du mois d'octobre les animaux se sont mis à maigrir de la même façon que ceux des troupeaux des éleveurs locaux.
Le poids moyen des femelles non suitées est passé de $383 \mathrm{~kg}$ le 15 octobre à 377 le 15 novembre, à 359 le 16 décembre et 349 le 3 janvier, soit en deux mois et demi une perte de poids de $34 \mathrm{~kg}$, donc de plus de 10 p. 100.

Début janvier, la feuillaison des Daniellia devenant importante, les animaux se sont immédiatement mis à l'exploiter sur les rejets, sur les arbustes et sur les parties des arbrisseaux qui leur étaient accessibles.

Chaque jour les animaux ont reçu $30 \mathrm{~kg}$ de feuilles fraîches soit $1,5 \mathrm{~kg}$ par animal; en 13 jours le poids total de l'ensemble du troupeau a légèrement augmenté : $7.128 \mathrm{~kg}$ le 15 janvier contre 7.097 le 3 janvier.

Les 4 dernières analyses du Tableau I donnent une idée de la valeur des aliments herbacés récoltables sur la savane par les animaux à cette époque de la saison sèche. L'analyse 3.933 représente ce que pourrait valoir la ration si les animaux consommaient les repousses et les pailles; l'analyse 4.890 correspond à un sérieux écrémage des bonnes parties de l'herbe donc à une récolte très faible quantitativement.

Les 2 autres analyses sont certainement plus proches de l'essentiel de la récolte des animaux car elles mesurent la valeur d'un mélange herbacé effectivement consommé par le bétail.

En reprenant les données proposées pour l'U.B.T. ("Unité bovin tropical») (5), l'animal de $250 \mathrm{~kg}$ en petits déplacements (le parc est de dimensions limitées) a besoin (entretien plus déplacements) de $2,7 \mathrm{UF}$ et $151 \mathrm{~g}$ de M.P.D. par jour. Ses possibilités d'ingestion sont d'environ $6,25 \mathrm{~kg}$ de matière sèche et sur ces bases, le gros de la ration récoltable apporterait 
3,5 UF, mais seulement $125 \mathrm{~g}$ de M.P.D. qui est alors incontestablement le facteur limitant. Or, nos feuilles de Daniellia parfaitement appétées et sans effort apportaient $5 \mathrm{UF}$ et $735 \mathrm{~g}$ de M.P.D. à l'équivalent de 28 U.B.T. de notre troupeau.

Les besoins énergétiques sont donc devenus excédentaires mais les $25 \mathrm{~g}$ de M.P.D. supplémentaires fournis permettent d'assurer les besoins azotés minimaux d'entretien.

En fait, ce qui est donné ici n'est qu'un schéma de ce qui se passe, car trop de facteurs mal connus interviennent et en particulier :

- Nos expériences d'ingestion de matière sèche en saison défavorable (12) nous ont donné avec ce genre d'aliment toujours moins que les $6,25 \mathrm{~kg}$ escomptés pour l'U.B.T.

- Par contre, avec une légumineuse bien appétée (Pueraria, Stylosanthes), nous nous approchions beaucoup de ces chiffres, et il est probable que le Daniellia oliveri favorise aussi une ingestion supérieure de M.S.

- L'amélioration de la ration protéinique augmente certainement la digestibilité générale de la ration de base.

- Enfin, les animaux ont sûrement absorbé un peu plus de feuilles que nous le disons, à cause de leur propre récolte, bien que les parties immédiatement accessibles aient en fait été très rapidement exploitées.

Il ressort en tout cas de ces observations que c'est essentiellement l'apport protéinique qui est à envisager en saison sèche, car la ration énergétique ne pose pas de gros problèmes pour autant que l'on dispose du fourrage sur pied.

\section{CONSEQUENCES PRATIQUES}

\section{a) Possibilités d'une complémentation}

On voit toute l'importance que peut prendre la pâture arborée en saison sèche, mais aussi combien il est facile de mal l'utiliser. Employée seule, elle est un énorme gaspillage d'azote, mais en complémentation, à dose juste suffisante, on risque de ne pas utiliser assez vite et au meilleur moment tout ce qui est intéressant. En tout cas, il importe de laisser aux animaux une pâture ad libitum d'herbe même sèche pendant les périodes de pâture arbustive pour que le meilleur parti en soit tiré; très vite et malgré la richesse certaine de l'aliment arbustif disponible, les possibilités limitées de récolte rendent cette ration elle aussi incomplète. C'est là un argument supplémentaire à ajouter à l'appui de la non mise à feu des parcs exploités en saison des pluies.

Il a également été montré tout l'intérêt que pouvait représenter l'intervention humaine dans l'exploitation optimale de cette précieuse source alimentaire. Cette intervention est hélas pratiquement inexistante dans l'Adamaoua.

\section{b) Traitement des espèces ligneuses}

En zone sahélienne, l'exploitation arbustive est courante mais extrêmement irrationnelle:

Les espèces appétées sont fréquemment ébranchées à hauteur d'homme. En parapluie, les branches incomplètement séparées du tronc retombent tout autour et permettent au premier feu une excellente «cuisson » de l'arbre qui en meurt très généralement, alors que cet élagage n'est pas en lui-même une catastrophe.

Que faudrait-il faire en Adamaoua pour permettre au bétail une meilleure exploitation de l'arbre?

Incontestablement, c'est un prélèvement aussi limité que celui opéré par les animaux qui serait souhaitable; c'est-à-dire un prélèvement qui lése au minimum les parties pérennes du végétal. C'est l'émondage qui s'en rapproche le plus. Pour les arbres et les arbrisseaux, il sera possible d'utiliser un échenulloir élagueur articulé, pour les parties inaccessibles. Toutefois, on peut envisager de former les sujets en place, soit pour tendre au taillis, soit pour obtenir des tétards de $1,20 \mathrm{~m}$ de haut environ. Les essais montrent que la constitution de cépées par coupe rez-terre n'est pas à envisager pour les grosses tiges qui, dès $15-20 \mathrm{~cm}$ de diamètre repartent de façon très aléatoire. Compte tenu également des dommages causés par le feu aux rejets et drageons, il vaut mieux former les jeunes sujets en tétards en empêchant les tiges de dépasser $1,20 \mathrm{~m}$ à $1,50 \mathrm{~m}$ de haut. Les vieux sujets acceptent volontiers de donner des repousses après avoir été coupés à $1,50 \mathrm{~m}$, mais la productivité diminue tellement qu'il vaut mieux essayer d'envisager un émondage. Cet émondage est certes plus difficile mais il permet 35 à $40 \mathrm{~kg}$ en plusieurs récoltes de matière 
verte dans la saison au lieu d'une seule de 20 à $25 \mathrm{~kg}$ au moment de la coupe, et seulement quelques $\mathrm{kg}$ les années suivantes.

\section{c) Absence de rotation en saison sèche}

Mais par dessus tout, pour tirer le meilleur parti de la pâture arborée naturellement prélevée par les animaux, il faut que ceux-ci soient en permanence au contact de la végétation ligneuse. Quelques jours d'absence et ce sera une partie de la pousse qui sera perdue sans possibilité d'exploitation des repousses qui pourraient se faire sur les parties déjà broutées. Cela souligne encore l'importance qu'il y a à ne pas manquer la première pâture possible. Il faut donc que les animaux soient en permanence dans les parcs qu'ils doivent exploiter, ce qui exclut toute notion de rotation pour les troupeaux, et convient d'ailleurs parfaitement à leur caractère vagabond.

\section{CONCLUSION GENERALE}

Peut-on conclure de cette étude à un dilemme opposant la savane pastorale à la savane forestière ? Certainement pas et ce qui précède incite plutôt à voir ces deux éléments comme fort heureusement complémentaires en ce qui concerne l'élevage dans ses conditions extensives actuelles. L'intensification qui conduit à une rationalisation de l'exploitation des espèces ligneuses appétées est obtenue par:

- l'élimination des espèces non appétées;

- la mise en forme des jeunes espèces appétées pour les rendre mieux exploitables directement par le bétail;

- La distribution au bétail des parties hors de son atteinte.

Cette utilisation de l'arbre est certainement la meilleure qui puisse être faite en Adamaoua, d'autant plus qu'elle est compatible avec une effective protection du sol contre les diverses formes de l'érosion.

Or, précisément la justification principale du maintien ou de la reconstitution de l'élément boisé est la nécessaire protection du sol et non pas la production de bois.

Son intérêt comme élément exploitable du pâturage est renforcé par le fait que son rendement en bois de feu est très faible; faible par sa productivité par hectare et par an proprement dite et faible surtout par le petit nombre des espèces exploitées pour le feu (Hymenocardia acida presque exclusivement). Par surcroît, les possibilités de production des reboisements artificiels en Adamaoua sont sans commune mesure avec celles de la végétation naturelle (Eucalyptus saligna ou $E$. grandis comme bois d'œuvre et de service sur sols riches, Cassia siamea à 30 stères/ha/an de bois de service et de feu).

Il faut aussi souligner la réalité d'une pâture arbustive en saison des pluies; c'est vraisemblablement elle qui interdit la mise en évidence d'une productivité animale meilleure sur les pâturages dessouchés que sur les pâturages intacts.

Il est important de ne pas dépasser certaines limites d'embroussaillement (environ 1.000 sujets/ha) où alors des espèces à couvert dense (et qui de plus ne sont pas appétées) s'installent et éliminent l'herbe, cependant que le feu est impuissant à les faire régresser tant qu'il y a pâture effective, celle-ci interdisant le passage de feux violents. Ces derniers, de plus, doivent intervenir plusieurs années consécutives pour atteindre la végétation ligneuse.

En définitive, le problème étant posé du maintien d'un équilibre convenable sylvopastoral, le reste est affaire d'aménagement et les solutions sont relativement simples. Passé ce stade de l'équilibre, le problème se complique et devient hors de portée du simple pasteur, car c'est un problème de régénération c'est-àdire :

- Si la vocation est pastorale; régénération de l'herbe, ce qui est fort complexe (débroussaillement au moins partiel, implantation d'espèces locales disparues, mises en défens, etc.).

- Si la vocation est forestière; soit maintenir et accroitre le couvert des espèces locales (ouverture des massifs boisés naturellement au parcours, politique de feu précoce au sens forestier du terme, etc.), soit remplacer ces derniers par les espèces à productivité élevée qui ont fait leurs preuves.

A plus ou moins longue échéance, l'arbre sera intégralement exclu des terrains de parcours. Mais alors des problèmes de complémentation du bétail en saison défavorable auront été résolus; de même on saura alors certainement 
nettoyer les terrains de parcours autrement que par le seul feu. En un mot, nous serons parvenus à un stade d'élevage intensif tout à fait possible dans une région où l'on peut tirer des pâturages naturels plus de 1.800 U.F. à l'hectare.

Mais en attendant, il faut utiliser au mieux l'herbe et l'arbre. Pour cela le débroussaillement sélectif est l'une des interventions élémentaires à vulgariser. L'application d'une politique rai- sonnée de mises à feu des pâturages est un autre facteur essentiel d'amélioration des conditions d'alimentation du bétail.

Le tout est de savoir si tous ces enseignements, que nous croyons fermement constituer " la bonne parole", peuvent être entendus dans le cadre d'une structure foncière trop imprécise au niveau de l'utilisateur.

\section{$\star \star$}

\section{SUMMARY}

"Woody pasture " in Cameroon. Use of ligneous shrubs by the cattle

The author relates observations, taken in pastures of Wakwa, near N'Gaoundere, concerning as well plants as cattle herds into the pasture. Feeding value of the species as their yield and cattle's behaviour are unfolded.

Practical inferences about possibilities of complementary feeding, treatment of the ligneous species, and necessity of keeping permanently the animals in the pastures, are drawn. In conclusion, the author points out that pastoral savannah and woodland savannah, for from being conflicting, are complementary in the conditions, now prevailing, of extensive animal husbandry and underlines the necessity of preserving an adequate sylvian-pastoral balance.

\section{RESUMEN}

\section{Pasto leñoso en Camerún. Su utilización por los bovinos}

El autor nota las observaciones efectuadas en los pastos de Wakwa cerca de N'Gaoundere sobre los plantones así como los lotes de animales al pasto. Se dan el valor bromatologico de las especies, su rendimiento y el comportamiento de los ganados.

Se evocan consecuencias prácticas en lo concerniente a las posibiJidades de suplementación, al tratamiento de las especies leñosas y a la necesidad de dejar en función continua los animales en los majadales.

En conclusión, el autor indica que la sabana de pasto y la sabana de selva no se oponen, al contrario son complementarias en las condiciones de la ganaderia extensiva actual y nota la necesidad del mantenimiento de un equilibrio silvo-pastoral conveniente.

\section{BIBLIOGRAPHIE}

1. AUBREVILLE (A.), «Flore forestière soudanoguinéenne », Paris, Soc. Ed. Géogr. Mar. et Col.. 1950.

2. AUBREVILLE (A.), «Flore forestière de Côted'Ivoire ", Nogent sur Marne, C.T.F.T., 1959 (Publ. no 15).

3. BILLE (J. C.), « Expérimentation agrostologique en République Centrafricaine », Maisons-Alfort, IEMVT, 1967 (Etude agrostologique $n^{*} 21$ ).

4. BOUDET (G.), «Etude agrostologique du Ranch de Sipilou (République de Côte d'Ivoire) ", Maisons-Alfort, I.E.M.V.T., 1966. (Etude agrostologique no 14).
5. BOUDET (G.), RIVIERE (R.), « Emploi pratique des analyses fourragères pour l'appréciation des pâturages tropicaux *, Rev. Elev. Méd. vét. Pays trop., 1968, 21 (2) : 227-66.

6. FERRANDO (R.), «Les bases de l'alimentation", Paris, Vigot Frères, 1964.

7. HUTCHINSON (J.), DALZIEL (J. M.), « Flora of West Tropical Afrika », London. Crown agents for ove"sea governments and administrations, 1954-1963.

8. LEROY, «L'Elevage rationnel des animaux domestiques », Tome I, alimentation. Paris, Hachette, 1965. 
9. MONNIER, PIOT (J.), “ Problèmes de pâturages dans l'Adamaoua », Bois Forêts Trop., 1964 (97) : 3-15, (98): 13-25.

10. PICCIONI (M.), « Dictionnaire des aliments pour les animaux », Bologna, Edagricole, 1965.

11. PIOT (J.), «Etudes pastorales en Adamaoua Camerounais", Rev. Elev. Méd. vét. Pays trop., 1966, 19 (1) : 45-61.
12. PIOT (J.), «Rapports Annuels. Station Fourragère de Wakwa 1965,-66 et 1966-67 ».

13. PIOT (J.), «Végétaux ligneux et pâturages des savanes de l'Adamaoua au Cameroun ", Rev. Elev. Méd. vét. Pays trop., 1969, 22 (4): 541-59.

14. SCHNEIDER (B.H.), «Feeds of the world. Their digestibility and composition $»$, Morgantown (U.S.A.), Agriculture experimental Station, 1947. 


\title{
NOMS SCIENTIFIQUES ET VERNACULAIRES (Baya) DES PRINCIPALES ESPECES LIGNEUSES DE LA STATION FOURRAGERE DE WAKWA
}

\author{
(Savane et partie Galeries forestières)
}

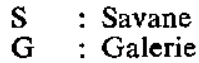

\author{
A : Appeté \\ TA : Très appeté \\ PA : Peu appeté \\ NA : Non appeté
}

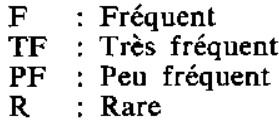

F : Fréquent

PF : Peu fréquent

R : Rare

\begin{tabular}{|c|c|c|c|c|c|}
\hline Noms scientifiques & Famille & $\begin{array}{l}\text { Nom } \\
\text { vernaculaire }\end{array}$ & Station & Appétib. & Fréq. \\
\hline $\begin{array}{l}\text { Acacia sieberiana var. villosa A. Chev. } \\
\text { Albizia coriaria Welw. } \\
\text { Albizia zygia J. F. Macbr. } \\
\text { Allophylus cf. grandifolius Rad. } \\
\text { Allophylus africanus P. Beauv. form. } \\
\text { africanus } \\
\text { Annona arenaria Thonn. } \\
\text { Anthocleista nobilis G. Don. } \\
\text { Antidesma venosum Tul. } \\
\text { Aubrevillea kerstingii Pellegr. } \\
\text { Bauhinia = Piliostigma } \\
\text { Beilschmiedia Spp. } \\
\text { Bombax buonopozense P. Beauv. } \\
\text { Borassus (flabellifer) aethiopum Mart. } \\
\text { Bridelia ferruginea Benth. } \\
\text { Bridelia ndellensis Beille } \\
\text { Bridelia cf. speciosa Müll. Arg. } \\
\text { Burkea africana Hook. } \\
\text { Butyrospermum paradoxum Hepper } \\
\text { var. parkii } \\
\text { Canthium venosum Hiern } \\
\text { Carissa edulis Vahl } \\
\text { Cassia petersiana Boll. } \\
\text { Clausena anisata Hook f. ex Benth. } \\
\text { Combretum nigricans et Comb. Sp. } \\
\text { Commiphora kerstingii Engl. } \\
\text { Craterispermum laurinum Benth. } \\
\text { Crossopteryx febrifuga (Afz.) Benth. } \\
\text { Croton macrostachyus Hochst. } \\
\text { Cussonia barteri Seemann } \\
\text { Daniellia oliveri Hutch. \& Dalz. } \\
\text { Deinbollia Sp. } \\
\text { Dombeya cf. multiflora } \\
\text { Ekebergia senegalensis A Juss. } \\
\text { Entada abyssinica Steud. } \\
\text { Entada africana Guill. \& Perr. } \\
\text { Eriocoelum kerstingii Gil. } \\
\text { Erythrina senegalensis DC. } \\
\text { Erythrina sigmoidea Hua } \\
\text { Eugenia Sp. } \\
\text { Fadogia erythrophloea Hutch. \& Dalz. } \\
\text { Fagara tessmanii Eng. } \\
\text { Faurea speciosa Welw. } \\
\text { Ficus capensis Thunb. } \\
\text { Ficus congensis Engl. } \\
\text { Ficus glumosa var. glaberrima Mart. } \\
\text { Ficus glumosa Del. } \\
\text { Ficus gnaphalocarpa Steud. } \\
\text { Ficus ovata Vahl } \\
\text { Ficus thonningii Blume } \\
\text { Ficus umbellata Vahl }\end{array}$ & $\begin{array}{l}\text { Mimosacées } \\
\text { Mimosacées } \\
\text { Mimosacées } \\
\text { Sapindacées } \\
\text { Sapindacées } \\
\text { Annonacées } \\
\text { Loganiacées } \\
\text { Euphorbiacées } \\
\text { Mimosacées } \\
\text { Casalpiniacées } \\
\text { Lauracées } \\
\text { Bombacacées } \\
\text { Arecacées } \\
\text { Euphorbiacées } \\
\text { Euphorbiacées } \\
\text { Euphorbiacées } \\
\text { Cæsalpiniacées } \\
\text { Sapotacées } \\
\text { Rubiacées } \\
\text { Apocynacées } \\
\text { Cæsalpiniacées } \\
\text { Rutacées } \\
\text { Combretacées } \\
\text { Burseracées } \\
\text { Rubiacées } \\
\text { Rubiacées } \\
\text { Euphorbiacées } \\
\text { Araliacées } \\
\text { Cæsalpiniacées } \\
\text { Sapindacées } \\
\text { Sterculiacées } \\
\text { Méliacées } \\
\text { Mimosacées } \\
\text { Mimosacées } \\
\text { Sapindacées } \\
\text { Papillionacées } \\
\text { Papillionacées } \\
\text { Myrtacées } \\
\text { Rubiacées } \\
\text { Rutacées } \\
\text { Proteacées } \\
\text { Moracées } \\
\text { Moracées } \\
\text { Moracées } \\
\text { Moracées } \\
\text { Moracées } \\
\text { Moracées } \\
\text { Moracées } \\
\text { Moracées }\end{array}$ & $\begin{array}{l}\text { Ngah } \\
\text { Tolla } \\
\text { Ndoya } \\
\text { Wi Norzer } \\
\text { Lossa } \\
\text { Soré } \\
\text { Zereforo } \\
\text { Boufibane } \\
\text { Soumbou } \\
\text { Domo } \\
\text { Ngala } \\
\text { Guerra } \\
\text { Koh } \\
\text { Sopo ou Nor } \\
\text { Norzer } \\
\text { Sopoli } \\
\text { Nbékéré } \\
\text { Kol } \\
\text { Ngazidila } \\
\text { Pinsela } \\
\text { Tefoto } \\
\text { Bathé } \\
\text { Pinsela } \\
\text { Goup } \\
\text { Foufouifou } \\
\text { Bogna } \\
\text { Kela ou Keha } \\
\text { Saj̈kabo } \\
\text { Soyi ou Sori } \\
\text { Henga } \\
\text { Nde-Nde } \\
\text { Nde-Nde } \\
\text { Ngekéré } \\
\text { Wi Borondong } \\
\text { Borondong } \\
\text { Wi-Zomo } \\
\text { Wi Kobo } \\
\text { Sototo ou Ngamnu } \\
\text { Tekoua } \\
\text { Mbora I } \\
\text { Tourou ou Batoui } \\
\text { Kolo } \\
\text { Kolo } \\
\text { Mbora } \\
\text { Balioko } \\
\text { Tui } \\
\text { Tourou } \\
\text { Tour }\end{array}$ & 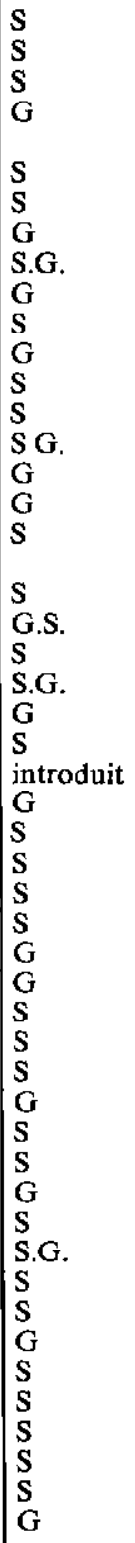 & $\begin{array}{l}\text { A } \\
\text { A } \\
\text { PA } \\
\text { TA }\end{array}$ & $\begin{array}{l}\text { PF } \\
\text { TF } \\
\text { PF } \\
\text { PF } \\
\text { PF } \\
\text { TF } \\
\text { R } \\
\text { R } \\
\text { PF } \\
\text { F } \\
\text { PF } \\
\text { PF } \\
\text { R } \\
\text { PF } \\
\text { PF } \\
\text { PF } \\
\text { R } \\
\text { F } \\
\text { F } \\
\text { PF } \\
\text { R } \\
\text { PF } \\
\text { F } \\
\text { TF } \\
\text { R } \\
\text { R } \\
\text { R } \\
\text { TF } \\
\text { PF } \\
\text { R } \\
\mathbf{R} \\
\mathbf{F} \\
\text { R } \\
\text { R } \\
\text { F } \\
\text { PF } \\
\text { PF } \\
\text { PF } \\
\text { PF } \\
\text { PF } \\
\text { F } \\
\text { PF } \\
\text { F } \\
\text { PF }\end{array}$ \\
\hline
\end{tabular}




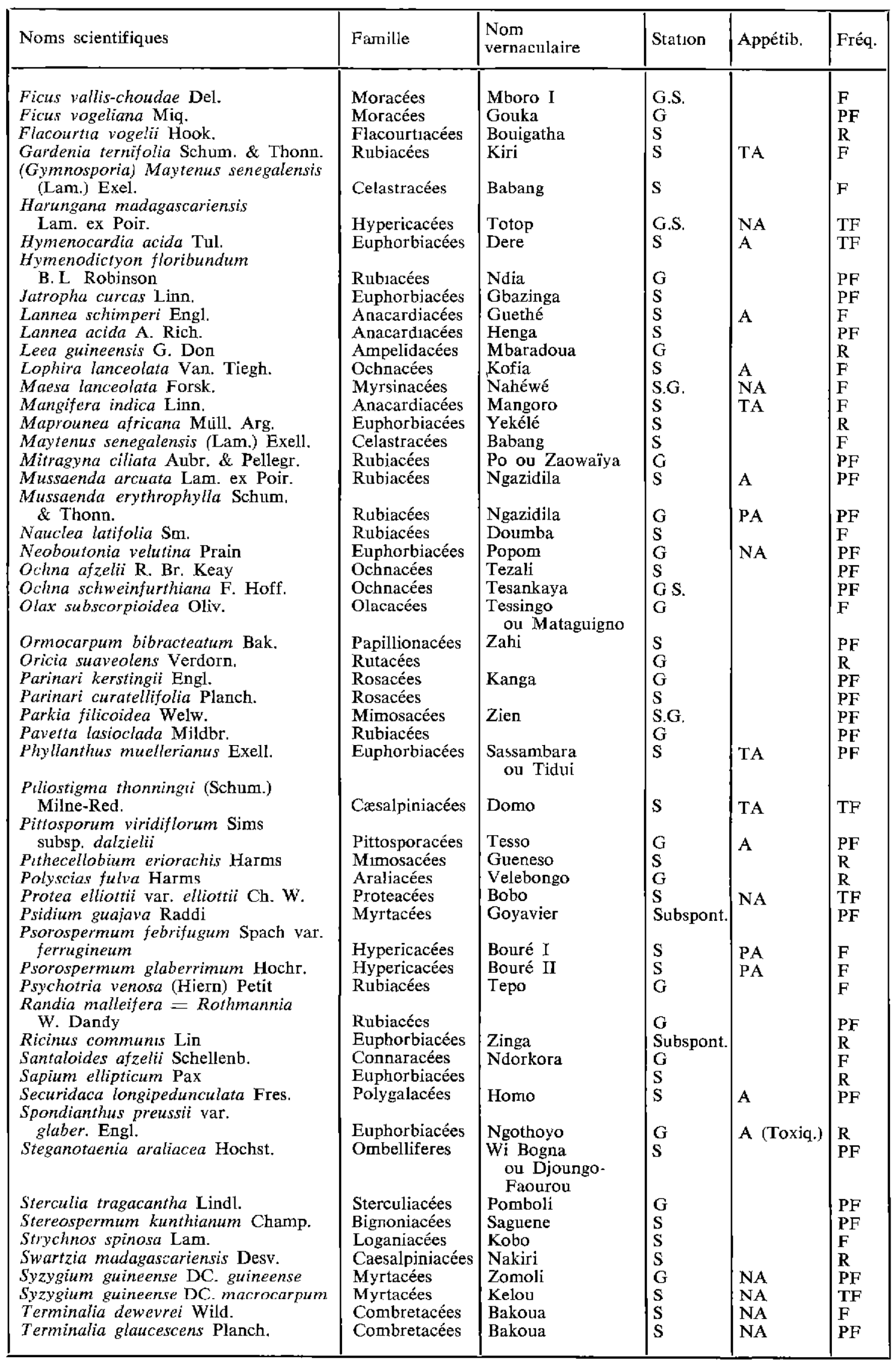




\begin{tabular}{|c|c|c|c|c|c|}
\hline Noms scientifiques & Famille & $\begin{array}{l}\text { Nom } \\
\text { vernaculaire }\end{array}$ & Station & Appetıb. & Ereq. \\
\hline $\begin{array}{l}\text { Termmalı macroptera Guill. \& Perr. } \\
\text { Trichlia roka Chiov. } \\
\text { Tricalysia okelensis var. oblanceolata } \\
\text { Uapaca togoensis Pax } \\
\text { Uvaria anonoides Bak. f. } \\
\text { Vernonia amygdalma Del. } \\
\\
\text { Vitex doniana Sweet. } \\
\text { Vitex madiensis Oliv. } \\
\text { Ximenia americana Linn. }\end{array}$ & $\begin{array}{l}\text { Combretacées } \\
\text { Meliacées } \\
\text { Rubiacées } \\
\text { Euphorbiacées } \\
\text { Anonacées } \\
\text { Composées } \\
\\
\text { Verbenacées } \\
\text { Verbenacées } \\
\text { Olacacées }\end{array}$ & $\begin{array}{l}\text { Bakoua } \\
\text { Pouyanga } \\
\text { Balatana } \\
\text { Dobo } \\
\text { Cor } \\
\text { Kassaka } \\
\text { Bakassaka ou } \\
\text { Ndolé } \\
\text { Bi ou Bili } \\
\text { Bilibetana } \\
\text { Mi ou Mili }\end{array}$ & $\begin{array}{l}S \\
S \\
G \\
G \\
G \\
S\end{array}$ & $\begin{array}{l}\text { PA } \\
\mathrm{A} \\
\mathrm{PA}\end{array}$ & $\begin{array}{l}\text { TF } \\
\text { PF } \\
F \\
\text { F } \\
\text { PF } \\
\text { F } \\
\\
\text { PF } \\
\text { PF } \\
\text { F }\end{array}$ \\
\hline
\end{tabular}

